\title{
Analytical Method of Modelling the Geometric System of Communication Route
}

\author{
Wladyslaw Koc \\ Faculty of Civil and Environmental Engineering, Gdansk University of Technology, Narutowicza 11/12, 80-233 Gdansk, Poland \\ Correspondence should be addressed to Wladyslaw Koc; kocwl@pg.gda.pl
}

Received 10 October 2013; Accepted 14 April 2014; Published 11 May 2014

Academic Editor: Youqing Wang

Copyright (C) 2014 Wladyslaw Koc. This is an open access article distributed under the Creative Commons Attribution License, which permits unrestricted use, distribution, and reproduction in any medium, provided the original work is properly cited.

The paper presents a new analytical approach to modelling the curvature of a communication route by making use of differential equations. The method makes it possible to identify both linear and nonlinear curvature. It enables us to join curves of the same or opposite signs of curvature. Solutions of problems for linear change of curvature and selected variants of nonlinear curvature in polynomial and trigonometric form were analyzed. A comparison of determined horizontal transition curves was made and examples of negotiating these curves into a geometric system were given.

\section{Introduction}

A rapid progress in computer calculation technique algorithms based on current knowledge and inspired by further prospecting is also taken into account in the field of geometrical shaping of communication routes. In the sphere of shaping the communication routes, vehicular roads and railway lines, the development of satellite measuring technique GNSS $[1,2]$ also plays a crucial role. A particular significance here will be attached to the question of vehicular dynamics. Therefore the modelling of curvature will obtain a key role.

This subject also includes the design of elements of the route of diversified curvature [3]. The elaboration of a method for a precise determination of the coordinate route points seems to be the most appropriate area of action. To perform this activity use is made of tools necessary to provide analytical solutions, that is, the most advantageous for practical application. The junction of elements of a vehicular route or railway line of diversified curvature should ensure a continuous change of the unbalanced side acceleration, in an advantageous way for interaction dynamics within the road-vehicle system. This is strictly connected with the proper shaping of the curvature.

The most developed investigation branch concerning this problem has been for years the study of transition curves connecting straight lengths of route with circular arcs. The problem of transition curves is related to vehicular roads and railway routes. However, it is easy to note that there is a distinct disproportion in taking interest in this problem. A search for new solutions is going on this sphere on vehicular roads (e.g., [4-12]). As regards the railway lines, the situation is entirely different. Publications on new transition curves are relatively rare and in the majority were published long ago (e.g., [13-17]).

The application of transition curves is aimed at ensuring a continuous change of the unbalanced side acceleration between intervals of motor road (or railway route) of diversified curvature in an advantageous way for the interaction dynamics within the road-vehicle system. Such a requirement relates to all sorts of transition curves. Under this situation it might appear that there is one particular algorithm to create them, common to all the analyzed curves. In fact most of the solutions that have been known so far appear independently and bear various names (sometimes coming from the name of their author). A general knowledge of the determination of transition curves equations would make it possible to compare various forms of curves with each other and to make an assessment of their practical application.

Twenty years ago this issue had already been sufficiently explained with respect mainly to railway routes [16]. Attention was then concentrated on working out a technique of identification of unbalanced accelerations occurring on various types of transition curves. It was based on a comparative analysis of some selected transition curves with the use of 
a dynamic model. In the method acceleration was a factor that excited the transverse vibrations of the carriage [18]. The main conclusion resulting from the considerations was to prove the relation existing between the response of the system and the class of the excitation function. The dynamic interactions were smaller (i.e., more advantageous) if the class of the function was higher. It turned out that evidently the largest acceleration values were noted on the cubical parabola (class of function $\mathrm{C}^{0}$ ). With respect to Bloss curve and a cosine curve (class $C^{1}$ ) they are significantly smaller, whereas on sine curve (class $\mathrm{C}^{2}$ ) they are the smallest.

After years the mentioned identification method of unbalanced accelerations [16] provided a source of inspiration for the elaboration of a new technique for modelling the geometric system of the communication route based on joining two points of the route with diversified curvature.

\section{Analytical Method of Modelling the Curvature}

A measure of the route bending is the ratio of the angle which determines the direction of the vehicle's longitudinal axis after covering a certain arc (Figure 1). The curvature of curve $K$ at point $M$ is called the boundary which is aimed at by the relation of acute angle $\Delta \Theta$ between tangents to curve $K$ at points $M$ and $M_{1}$, to the length of $\operatorname{arc} \Delta l$ when point $M_{1}$ tends along curve $K$ to point $M$

$$
k=\lim _{\Delta l \rightarrow 0}\left|\frac{\Delta \Theta}{\Delta l}\right|=\frac{d \Theta}{d l} .
$$

If the operation procedure by the use of rectangular coordinates $x, y$ is to be continued, it is necessary to take into consideration the sign of the curvature. As illustrated in Figure 1 angle $\Delta \Theta=\Theta_{1}-\Theta>0$; the curvature is assumed here that it has a positive value which, as can be seen, accompanies the curves with downward convexities. A negative value of curvature corresponds to angle $\Delta \Theta<0$ and appears on curves of an upward convexity.

Making a generalization of the identification method applied to unbalanced accelerations occurring on various types of transition curves [16], it is possible to search for curvature function $k(l)$ among the solutions of the differential equation

$$
k^{(m)}(l)=f\left[l, k, k^{\prime}, \ldots, k^{(m-1)}\right]
$$

with conditions for the transition curve at the outset (for $l=$ 0 ) and at the end (for $l=l_{k}$ )

$$
\begin{aligned}
k^{(i)}\left(0^{+}\right) & = \begin{cases}k_{1} & \text { for } i=0 \\
0 & \text { for } i=1,2, \ldots, n_{1},\end{cases} \\
k^{(j)}\left(l_{k}^{-}\right) & = \begin{cases}k_{2} & \text { for } j=0 \\
0 & \text { for } j=1,2, \ldots, n_{2},\end{cases}
\end{aligned}
$$

where $k_{1}$ and $k_{2}$ indicate the curvature magnitudes on both ends of the curve (taking note of an adequate sign). The order of the differential equation (2) is $m=n_{1}+n_{2}+2$, and

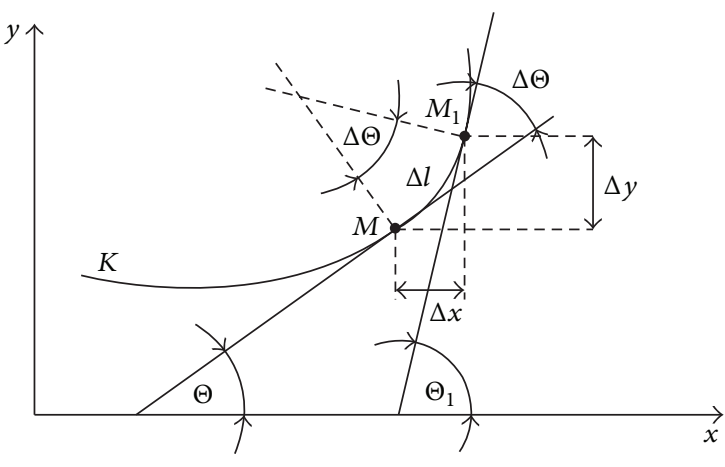

FIGURE 1: Schematic diagram for the explanation of the notion of curvature.

the obtained function $k(l)$ is of class $\mathrm{C}^{n}$ in the range $\left\langle 0, l_{k}\right\rangle$, where $n=\min \left(n_{1}, n_{2}\right)$.

The application of the method under consideration makes it possible to combine various geometric elements, for example, a straight with a circular arc, and also, after introducing an adequate sign of curvature, two circular arcs of a consistent run or converse arcs.

On determining the curvature function $k(l)$ a fundamental task is to find the coordinates that correspond to a curve in the rectangular coordinate system $x, y$. A solution for such a system is required by the satellite measuring technique GNSS [19-21], which becomes an aid in the determination of the coordinate points in a uniform, 3D-system of coordinates WGS 84 (the world geodetic system 1984). Next the measured ellipsoidal coordinates (GPS) are transformed to Gauss$\operatorname{Kruger}(X, Y)$ conformal coordinates [22]. An assumption is made that the beginning of system $x, y$ is at the terminal point of the input curve (with curvature $k_{1}$ ), while the axis of abscissae is tangent to this curve at this point.

The equation of the sought after connection can be written in parametric form

$$
\begin{aligned}
& x(l)=\int \cos \Theta(l) d l, \\
& y(l)=\int \sin \Theta(l) d l .
\end{aligned}
$$

Parameter $l$ is the position of a given point along the curve length. Function $\Theta(l)$ is determined on the basis of the formula

$$
\Theta(l)=\int k(l) d l
$$

The presented method of modelling the curvature has a universal character. It can be applied to both vehicular roads and railway routes. In the case of railway routes there arises an additional possibility, for modelling in a similar way, the superelevation ramp, understood here as a determined difference of heights of the rail. The principle of operation is similar to curvature modelling. The only differences are that $k_{1}$ has to be replaced by the value of superelevation $h_{1}$ at the beginning of the superelevation ramp and $k_{2}$ has to be replaced by the value of superelevation $h_{2}$ at the end of the superelevation 
ramp. The ramp length, of course, corresponds to the length of the transition curve. Using the same procedure it is possible to find the unbalanced acceleration $a(l)$.

\section{Transition Curve of Linear Curvature}

3.1. Finding Out the Curvature Equation. Linear change of curvature along a defined length $l_{k}$ is obtained by assuming two principal conditions

$$
\begin{aligned}
& k\left(0^{+}\right)=k_{1}, \\
& k\left(l_{k}^{-}\right)=k_{2},
\end{aligned}
$$

and a differential equation

$$
k^{\prime \prime}(l)=0 .
$$

After the determination of constants the solution of the differential problem (6), (7) is as follows:

$$
k(l)=k_{1}+\frac{1}{l_{k}}\left(k_{2}-k_{1}\right) l .
$$

Function $\Theta(l)$ is obtained from formula (5). In the case under consideration

$$
\Theta(l)=k_{1} l+\frac{1}{2 l_{k}}\left(k_{2}-k_{1}\right) l^{2} .
$$

3.2. The Determination of the Transition Curve Length (for Railway Lines). With regard to rail tracks the transition curve must satisfy two kinematic conditions

$$
\begin{gathered}
\psi_{\max } \leq \psi_{\text {dop }} \\
f_{\max } \leq f_{\text {dop }}
\end{gathered}
$$

where $\psi_{\max }$ is the maximum value of acceleration increment on transition curve in $\mathrm{m} / \mathrm{s}^{3}, \psi_{\text {dop }}$ is permissible value of acceleration increment in $\mathrm{m} / \mathrm{s}^{3}, f_{\max }$ is the maximum speed of lifting the wheel on superelevation ramp in $\mathrm{mm} / \mathrm{s}$, and $f_{\text {dop }}$ is permissible value of speed of lifting the wheel in $\mathrm{mm} / \mathrm{s}$.

The unbalanced acceleration $a(l)$ occurring on the transition curve results from speed $v$ of the train, magnitude of curvature, and ordinates of the superelevation ramp; its course is similar to $k(l)$ and $h(l)$. Analogously with (8)

$$
a(l)=a_{1}+\frac{1}{l_{k}}\left(a_{2}-a_{1}\right) l,
$$

where $a_{1}$ and $a_{2}$ are accelerations at ends of the transition curve

$$
a_{1}=\frac{v^{2}}{\operatorname{rad}} k_{1}-g \frac{h_{1}}{s}, \quad a_{2}=\frac{v^{2}}{\operatorname{rad}} k_{2}-g \frac{h_{2}}{s}
$$

( $h_{1}$ and $h_{2}$ are ordinates of the superelevation ramp at its ends).

On the assumption that the speed of the train $v=$ const., the formula for acceleration increment $\psi(l)$ is as follows:

$$
\psi(l)=v \frac{d}{d l} a(l)=\frac{v}{l_{k}}\left(a_{2}-a_{1}\right) .
$$

As can be seen the acceleration increment is here a constant value. However, from condition (10) it follows that

$$
l_{k}^{\psi} \geq \frac{v}{\psi_{\text {dop }}}\left|a_{2}-a_{1}\right|
$$

The superelevation ramp equation in the case under consideration is similar to (8) and (12)

$$
h(l)=h_{1}+\frac{1}{l_{k}}\left(h_{2}-h_{1}\right) l
$$

The speed of lifting the wheel $f(l)$ on the superelevation ramp (assuming that the travelling speed of the train $v=$ const.) is determined by the equation

$$
f(l)=v \frac{d}{d l} h(l)=\frac{v}{l_{k}}\left(h_{2}-h_{1}\right)
$$

Thus, also $f(l)$ is here a constant value, but from condition (11) it follows that

$$
l_{k}^{f} \geq \frac{v}{f_{\text {dop }}}\left|h_{2}-h_{1}\right|
$$

The assumed length of the transition curve must fulfill the condition

$$
l_{k} \geq \max \left(l_{k}^{\psi}, l_{k}^{f}\right)
$$

3.3. Coordinates of Transition Curve Connecting Uniform Curvatures. The determination of $x(l)$ and $y(l)$ by using (4) will need the expansion of integrands into Maclaurin series. After completing the whole procedure the following parametric equations are obtained:

$$
\begin{aligned}
x(l)= & \int \cos \Theta(l) d l \\
= & l-\frac{k_{1}^{2}}{6} l^{3}-\frac{k_{1}}{8 l_{k}}\left(k_{2}-k_{1}\right) l^{4} \\
& +\left[\frac{k_{1}^{4}}{120}-\frac{1}{40 l_{k}^{2}}\left(k_{2}-k_{1}\right)^{2}\right] l^{5}+\frac{k_{1}^{3}}{72 l_{k}}\left(k_{2}-k_{1}\right) l^{6} \\
& -\left[\frac{k_{1}^{6}}{5040}-\frac{k_{1}^{2}}{112 l_{k}^{2}}\left(k_{2}-k_{1}\right)^{2}\right] l^{7} \\
& -\left[\frac{k_{1}^{5}}{1920 l_{k}}\left(k_{2}-k_{1}\right)-\frac{k_{1}}{384 l_{k}^{3}}\left(k_{2}-k_{1}\right)^{3}\right] l^{8} \\
& +\left[\frac{k_{1}^{8}}{362880}-\frac{k_{1}^{4}}{1728 l_{k}^{2}}\left(k_{2}-k_{1}\right)^{2}\right. \\
& \left.+\frac{1}{3456 l_{k}^{4}}\left(k_{2}-k_{1}\right)^{4}\right] l^{9} \ldots
\end{aligned}
$$




$$
\begin{aligned}
y(l)= & \int \sin \Theta(l) d l \\
= & \frac{k_{1}}{2} l^{2}+\frac{1}{6 l_{k}}\left(k_{2}-k_{1}\right) l^{3}-\frac{k_{1}^{3}}{24} l^{4}-\frac{k_{1}^{2}}{20 l_{k}}\left(k_{2}-k_{1}\right) l^{5} \\
& +\left[\frac{k_{1}^{5}}{720}-\frac{k_{1}}{48 l_{k}^{2}}\left(k_{2}-k_{1}\right)^{2}\right] l^{6} \\
& +\left[\frac{k_{1}^{4}}{336 l_{k}}\left(k_{2}-k_{1}\right)-\frac{1}{336 l_{k}^{3}}\left(k_{2}-k_{1}\right)^{3}\right] l^{7} \\
& -\left[\frac{k_{1}^{7}}{40320}-\frac{k_{1}^{3}}{384 l_{k}^{2}}\left(k_{2}-k_{1}\right)^{2}\right] l^{8} \\
& -\left[\frac{k_{1}^{6}}{12960 l_{k}}\left(k_{2}-k_{1}\right)-\frac{k_{1}^{2}}{864 l_{k}^{3}}\left(k_{2}-k_{1}\right)^{3}\right] l^{9}+\cdots .
\end{aligned}
$$

Equations (20) for $k_{1}=0$ describe the curve in the form of clothoid used to connect a straight with a circular arc.

\subsection{Coordinates of Transition Curve Connecting Inverse Cur-} vatures. At inverse arcs (i.e., with diversified curvature signs) there arises the problem related to the determined coordinates $x(l)$ and $y(l)$, due to the course of function $\Theta(l)$. For $\operatorname{sign} k_{1}=\operatorname{sign} k_{2}$ function $\Theta(l)$ is a monotonic function, whereas for $\operatorname{sign} k_{1} \neq \operatorname{sign} k_{2}$ in the diagram of function $\Theta(l)$ there appears an extremum at point $l_{0}$, where

$$
\Theta^{\prime}\left(l_{0}\right)=k\left(l_{0}\right)=k_{1}+\left(k_{2}-k_{1}\right) \frac{l_{0}}{l_{k}}=0 .
$$

Values $l_{0}$ and $\Theta\left(l_{0}\right)$ follow from the relations:

$$
\begin{gathered}
l_{0}=-\frac{k_{1}}{k_{2}-k_{1}} l_{k} \\
\Theta\left(l_{0}\right)=-\frac{k_{1}^{2}}{2\left(k_{2}-k_{1}\right)} l_{k} .
\end{gathered}
$$

Figure 2 illustrates a scheme of angle $\Theta(l)$ for the connection of inverse circular arcs of radii $R_{1}=1200 \mathrm{~m}$ and $R_{2}=700 \mathrm{~m}$. The length of the transition curve $l_{k}=60 \mathrm{~m}$ was determined for speed value $v=90 \mathrm{~km} / \mathrm{h}$ using the procedure as described at Section 3.2 (the assumed superelevation values were $h_{1}=20 \mathrm{~mm}$ and $h_{2}=45 \mathrm{~mm}$ ).

In this situation the parametric equations of transition curve (20) are effective for $l \in\left\langle 0, l_{0}\right\rangle$; for $l \in\left(l_{0}, l_{k}\right\rangle$ functions

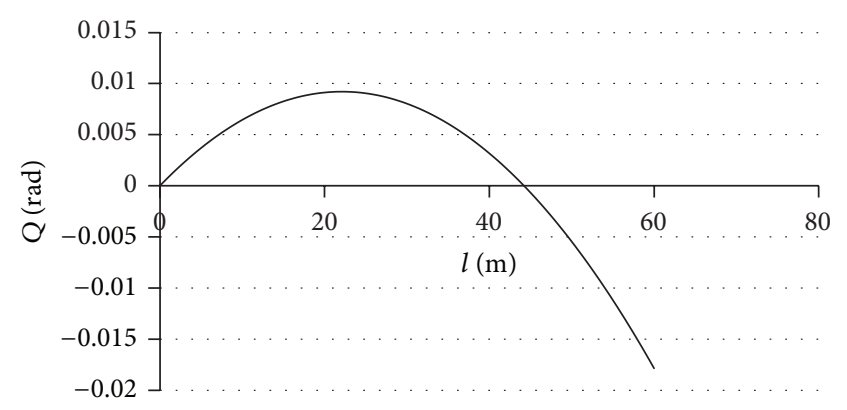

Figure 2: Diagram of angle $\Theta(l)$ defined by (9) for circular arcs connection of curvatures $k_{1}=1 / 1200 \mathrm{rad} / \mathrm{m}$ and $k_{2}=$ $-1 / 700 \mathrm{rad} / \mathrm{m}$ by means of transition curve of length $l_{k}=60 \mathrm{~m}$.

$\cos \Theta(l)$ and $\sin \Theta(l)$ should be expanded to Taylor series. After integration of the equations take the following form:

$$
\begin{aligned}
& x(l)=\int \cos \Theta(l) d l \\
& =x\left(l_{0}\right)+\cos \left(-\frac{1}{2} \frac{k_{1}^{2}}{k_{2}-k_{1}} l_{k}\right)\left(l-l_{0}\right) \\
& -\left[\frac{1}{6 l_{k}}\left(k_{2}-k_{1}\right) \sin \left(-\frac{1}{2} \frac{k_{1}^{2}}{k_{2}-k_{1}} l_{k}\right)\right]\left(l-l_{0}\right)^{3} \\
& -\left[\frac{1}{40 l_{k}^{2}}\left(k_{2}-k_{1}\right)^{2} \cos \left(-\frac{1}{2} \frac{k_{1}^{2}}{k_{2}-k_{1}} l_{k}\right)\right]\left(l-l_{0}\right)^{5} \\
& +\left[\frac{1}{336 l_{k}^{3}}\left(k_{2}-k_{1}\right)^{3} \sin \left(-\frac{1}{2} \frac{k_{1}^{2}}{k_{2}-k_{1}} l_{k}\right)\right]\left(l-l_{0}\right)^{7} \\
& +\cdots \\
& y(l)=\int \sin \Theta(l) d l \\
& =y\left(l_{0}\right)+\sin \left(-\frac{1}{2} \frac{k_{1}^{2}}{k_{2}-k_{1}} l_{k}\right)\left(l-l_{0}\right) \\
& +\left[\frac{1}{6 l_{k}}\left(k_{2}-k_{1}\right) \cos \left(-\frac{1}{2} \frac{k_{1}^{2}}{k_{2}-k_{1}} l_{k}\right)\right]\left(l-l_{0}\right)^{3} \\
& -\left[\frac{1}{40 l_{k}^{2}}\left(k_{2}-k_{1}\right)^{2} \sin \left(-\frac{1}{2} \frac{k_{1}^{2}}{k_{2}-k_{1}} l_{k}\right)\right]\left(l-l_{0}\right)^{5} \\
& -\left[\frac{1}{336 l_{k}^{3}}\left(k_{2}-k_{1}\right)^{3} \cos \left(-\frac{1}{2} \frac{k_{1}^{2}}{k_{2}-k_{1}} l_{k}\right)\right]\left(l-l_{0}\right)^{7} \\
& +\left[\frac{1}{3456 l_{k}^{4}}\left(k_{2}-k_{1}\right)^{4} \sin \left(-\frac{1}{2} \frac{k_{1}^{2}}{k_{2}-k_{1}} l_{k}\right)\right]\left(l-l_{0}\right)^{9} \\
& +\cdots \text {. }
\end{aligned}
$$


On completing the parametric equations (20) and (23) along with (24) a correct solution of the task for the case of diversified curvature signs is obtained (Figure 3). From a practical point of view the determination of the magnitude of the tangent slope angle at the end of the transition curve is very important. It amounts to

$$
\Theta\left(l_{k}\right)=\frac{1}{2}\left(k_{1}+k_{2}\right) l_{k} .
$$

A knowledge of $\Theta\left(l_{k}\right)$ makes it possible to add the transition curve to the other one having curvature $k_{2}$ at its initial point (satisfying the tangency condition of both the curves).

\section{Transition Curve of Polynomial Curvature}

From the point of view of vehicles' dynamics, the transition curve of linear change of curvature is not the most advantageous solution [23]. A definitely better one is nonlinear solution whose characteristics are interesting enough to be compared with the Bezier curves preferable lately in literature $[4,7]$.

The differential equation (2) allows to appoint an unlimited number of curves with nonlinear curvature change. In the case of geometric layout of communication routes it will be appropriate to consider the transition curves of polynomial and trigonometric curvature. In fact such forms of curves are used in the standard ways to connect a straight line with a circular arc.

4.1. Formulation of the Curvature Equation. To formulate the curvature equation in the polynomial form, let us assume the following boundary conditions:

$$
\begin{array}{ll}
k\left(0^{+}\right)=k_{1}, & k\left(l_{k}^{-}\right)=k_{2} \\
k^{\prime}\left(0^{+}\right)=0, & k^{\prime}\left(l_{k}^{-}\right)=0
\end{array}
$$

and the differential equation

$$
k^{(4)}(l)=0 .
$$

After solving the differential problem (26), (27) the following formula for curvature $k(l)$ is obtained:

$$
k(l)=k_{1}+\frac{3}{l_{k}^{2}}\left(k_{2}-k_{1}\right) l^{2}-\frac{2}{l_{k}^{3}}\left(k_{2}-k_{1}\right) l^{3} .
$$

The form of function $\Theta(l)$ determined on the basis of (5) and (28) is as follows:

$$
\Theta(l)=k_{1} l+\frac{1}{l_{k}^{2}}\left(k_{2}-k_{1}\right) l^{3}-\frac{1}{2 l_{k}^{3}}\left(k_{2}-k_{1}\right) l^{4} .
$$

On rail routes the length of the transition curve is determined like at Section 3.2 for solution of linear curvature. The following conditions should be fulfilled here:

$$
\begin{aligned}
& l_{k}^{\psi} \geq \frac{3}{2} \frac{v}{\psi_{\text {dop }}}\left|a_{2}-a_{1}\right|, \\
& l_{k}^{f} \geq \frac{3}{2} \frac{v}{f_{\text {dop }}}\left|h_{2}-h_{1}\right| .
\end{aligned}
$$

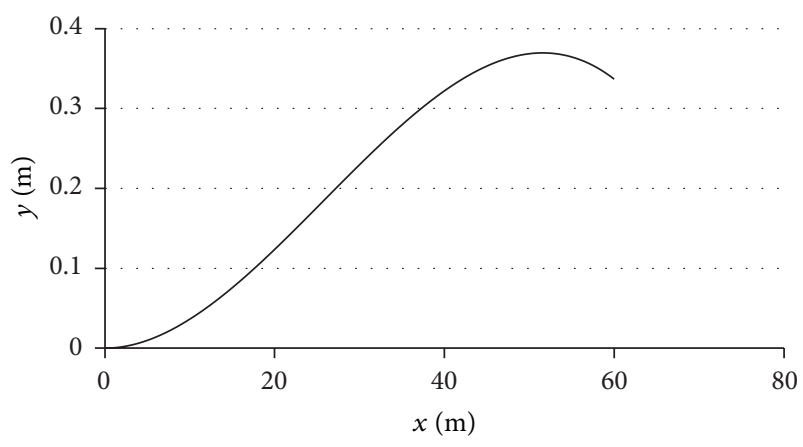

FIgURE 3: Diagram of horizontal ordinates $y(x)$ defined by (20), (23), and (24) for the joint of circular arcs of curvatures $k_{1}=$ $1 / 1200 \mathrm{rad} / \mathrm{m}$ and $k_{2}=-1 / 700 \mathrm{rad} / \mathrm{m}$ with transition curve of length $l_{k}=60 \mathrm{~m}$ (using different horizontal and vertical scales).

The adopted length of the transition curve must satisfy condition (19).

4.2. Coordinates of Transition Curve Connecting Uniform Curvatures. On expanding functions $\cos \Theta(l)$ and $\sin \Theta(l)$ into Maclaurin series and after integration, the following parametric equations are acquired:

$$
\begin{aligned}
& x(l)=\int \cos \Theta(l) d l \\
& =l-\frac{k_{1}^{2}}{6} l^{3}+\left[\frac{k_{1}^{4}}{120}-\frac{k_{1}}{5 l_{k}^{2}}\left(k_{2}-k_{1}\right)\right] l^{5} \\
& +\left[\frac{k_{1}}{12 l_{k}^{3}}\left(k_{2}-k_{1}\right)\right] l^{6} \\
& -\left[\frac{k_{1}^{6}}{5040}-\frac{k_{1}^{3}}{42 l_{k}^{2}}\left(k_{2}-k_{1}\right)+\frac{1}{14 l_{k}^{4}}\left(k_{2}-k_{1}\right)^{2}\right] l^{7} \\
& -\left[\frac{k_{1}^{3}}{96 l_{k}^{3}}\left(k_{2}-k_{1}\right)-\frac{1}{16 l_{k}^{5}}\left(k_{2}-k_{1}\right)^{2}\right] l^{8} \\
& +\left[\frac{k_{1}^{8}}{362880}-\frac{k_{1}^{5}}{1080 l_{k}^{2}}\left(k_{2}-k_{1}\right)+\frac{k_{1}^{2}}{36 l_{k}^{4}}\left(k_{2}-k_{1}\right)^{2}\right. \\
& \left.+\frac{1}{72 l_{k}^{6}}\left(k_{2}-k_{1}\right)^{2}\right] l^{9}+\cdots \\
& y(l)=\int \sin \Theta(l) d l \\
& =\frac{k_{1}}{2} l^{2}-\left[\frac{k_{1}^{3}}{24}-\frac{1}{4 l_{k}^{2}}\left(k_{2}-k_{1}\right)\right] l^{4} \\
& -\frac{1}{10 l_{k}^{3}}\left(k_{2}-k_{1}\right) l^{5}+\left[\frac{k_{1}^{5}}{720}-\frac{k_{1}^{2}}{12 l_{k}^{2}}\left(k_{2}-k_{1}\right)\right] l^{6} \\
& +\frac{k_{1}^{2}}{28 l_{k}^{3}}\left(k_{2}-k_{1}\right) l^{7}
\end{aligned}
$$




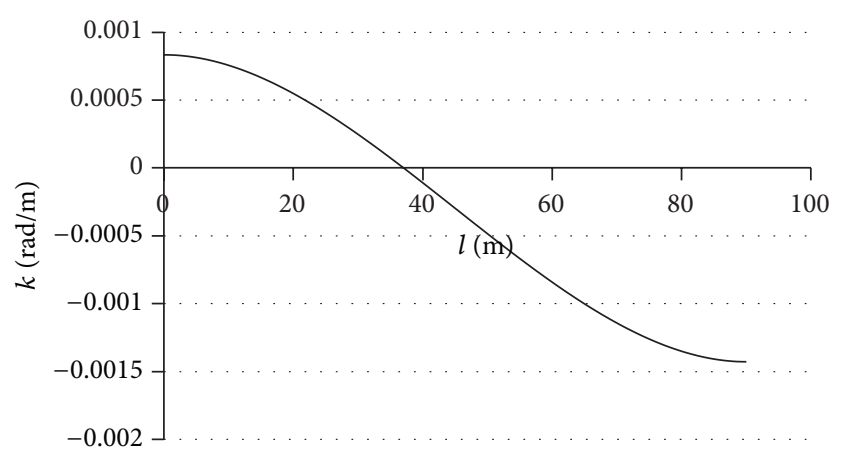

FIgURE 4: Diagram of curvature $k(l)$ described by (28) for the connection of circular arcs of curvatures $k_{1}=1 / 1200 \mathrm{rad} / \mathrm{m}$ and $k_{2}=-1 / 700 \mathrm{rad} / \mathrm{m}$ with the use of transition curve of length $l_{k}=$ $90 \mathrm{~m}$.

$$
\begin{aligned}
& -\left[\frac{k_{1}^{7}}{40320}-\frac{k_{1}^{4}}{192 l_{k}^{2}}\left(k_{2}-k_{1}\right)+\frac{k_{1}}{16 l_{k}^{4}}\left(k_{2}-k_{1}\right)^{2}\right] l^{8} \\
& +\left[\frac{k_{1}}{18 l_{k}^{5}}\left(k_{2}-k_{1}\right)^{2}-\frac{k_{1}^{4}}{432 l_{k}^{3}}\left(k_{2}-k_{1}\right)\right] l^{9}+\cdots
\end{aligned}
$$

Equations (31) for $k_{1}=0$ describe the so-called Bloss curve which can be used to join a straight to a circular arc.

4.3. Coordinates of Transition Curve Connecting Inverse Curvatures. When the arcs are inverse (i.e., the curvature signs are different, Figure 4) there occurs a problem relating to the determined coordinates $x(l)$ and $y(l)$ which results from function $\Theta(l)$. For $\operatorname{sign} k_{1}=\operatorname{sign} k_{2}$ function $\Theta(l)$ is a monotonic function, while for $\operatorname{sign} k_{1} \neq \operatorname{sign} k_{2}$ in the diagram of function $\Theta(l)$ there appears extremum at point $l_{0}$, where

$$
\Theta^{\prime}\left(l_{0}\right)=k\left(l_{0}\right)=k_{1}+\frac{3}{l_{k}^{2}}\left(k_{2}-k_{1}\right) l_{0}^{2}-\frac{2}{l_{k}^{3}}\left(k_{2}-k_{1}\right) l_{0}^{3}=0
$$

Parametric equations of transition curve (31) are valid for $l \in\left\langle 0, l_{0}\right\rangle$. The determination of value $l_{0}$ makes it necessary to solve (32) which is cubic. The sought-after value $l_{0} \in\left\langle 0, l_{k}\right\rangle$, satisfying conditions of the problem is defined by the formula

$$
l_{0}=\left[\frac{1}{2}-\cos \left(\frac{\varphi}{3}+\frac{\pi}{3}\right)\right] l_{k}
$$

where angle $\varphi$ follows from the relation

$$
\cos \varphi=\frac{k_{1}+k_{2}}{k_{2}-k_{1}}
$$

Under such circumstances in order to determine parametric equations of the transition curve for $l \in\left(l_{0}, l_{k}\right\rangle$, functions $\cos \Theta(l)$ and $\sin \Theta(l)$ should be expanded into Taylor series; after integration the following parametric equations of the transition curve are obtained:

$$
\begin{aligned}
& x(l)=\int \cos \Theta(l) d l \\
& =x\left(l_{0}\right)+\cos \Theta\left(l_{0}\right)\left(l-l_{0}\right) \\
& -\frac{1}{6} \sin \Theta\left(l_{0}\right) \Theta^{\prime \prime}\left(l_{0}\right)\left(l-l_{0}\right)^{3} \\
& -\frac{1}{24} \sin \Theta\left(l_{0}\right) \Theta^{\prime \prime \prime}\left(l_{0}\right)\left(l-l_{0}\right)^{4} \\
& -\left\{\frac{1}{40} \cos \Theta\left(l_{0}\right)\left[\Theta^{\prime \prime}\left(l_{0}\right)\right]^{2}\right. \\
& \left.+\frac{1}{120} \sin \Theta\left(l_{0}\right) \Theta^{(4)}\left(l_{0}\right)\right\}\left(l-l_{0}\right)^{5} \\
& -\frac{1}{72} \cos \Theta\left(l_{0}\right) \Theta^{\prime \prime}\left(l_{0}\right) \Theta^{\prime \prime \prime}\left(l_{0}\right)\left(l-l_{0}\right)^{6} \\
& +\left\{\frac{1}{336} \sin \Theta\left(l_{0}\right)\left[\Theta^{\prime \prime}\left(l_{0}\right)\right]^{3}\right. \\
& -\frac{1}{336} \cos \Theta\left(l_{0}\right) \Theta^{\prime \prime}\left(l_{0}\right) \Theta^{(4)}\left(l_{0}\right) \\
& \left.-\frac{1}{504} \cos \Theta\left(l_{0}\right)\left[\Theta^{\prime \prime \prime}\left(l_{0}\right)\right]^{2}\right\}\left(l-l_{0}\right)^{7} \\
& +\left\{\frac{1}{384} \sin \Theta\left(l_{0}\right)\left[\Theta^{\prime \prime}\left(l_{0}\right)\right]^{2} \Theta^{\prime \prime \prime}\left(l_{0}\right)\right. \\
& \left.-\frac{1}{1152} \cos \Theta\left(l_{0}\right) \Theta^{\prime \prime \prime}\left(l_{0}\right) \Theta^{(4)}\left(l_{0}\right)\right\}\left(l-l_{0}\right)^{8} \\
& +\left\{\frac{1}{3456} \cos \Theta\left(l_{0}\right)\left[\Theta^{\prime \prime}\left(l_{0}\right)\right]^{4}\right. \\
& +\frac{1}{1296} \sin \Theta\left(l_{0}\right) \Theta^{\prime \prime}\left(l_{0}\right)\left[\Theta^{\prime \prime \prime}\left(l_{0}\right)\right]^{2} \\
& +\frac{1}{1728} \sin \Theta\left(l_{0}\right)\left[\Theta^{\prime \prime}\left(l_{0}\right)\right]^{2} \Theta^{(4)}\left(l_{0}\right) \\
& \left.-\frac{1}{10368} \cos \Theta\left(l_{0}\right)\left[\Theta^{(4)}\left(l_{0}\right)\right]^{2}\right\}\left(l-l_{0}\right)^{9}+\cdots
\end{aligned}
$$$$
y(l)=\int \sin \Theta(l) d l
$$$$
=y\left(l_{0}\right)+\sin \Theta\left(l_{0}\right)\left(l-l_{0}\right)
$$$$
+\frac{1}{6}\left[\cos \Theta\left(l_{0}\right) \Theta^{\prime \prime}\left(l_{0}\right)\right]\left(l-l_{0}\right)^{3}
$$$$
+\frac{1}{24}\left[\cos \Theta\left(l_{0}\right) \Theta^{\prime \prime \prime}\left(l_{0}\right)\right]\left(l-l_{0}\right)^{4}
$$$$
-\left\{\frac{1}{40} \sin \Theta\left(l_{0}\right)\left[\Theta^{\prime \prime}\left(l_{0}\right)\right]^{2}\right.
$$$$
\left.-\frac{1}{120} \cos \Theta\left(l_{0}\right) \Theta^{(4)}\left(l_{0}\right)\right\}\left(l-l_{0}\right)^{5}
$$$$
-\frac{1}{72}\left[\sin \Theta\left(l_{0}\right) \Theta^{\prime \prime}\left(l_{0}\right) \Theta^{\prime \prime \prime}\left(l_{0}\right)\right]\left(l-l_{0}\right)^{6}
$$ 


$$
\begin{aligned}
&-\left\{\frac{1}{336} \cos \Theta\left(l_{0}\right)\left[\Theta^{\prime \prime}\left(l_{0}\right)\right]^{3}\right. \\
&+\frac{1}{336} \sin \Theta\left(l_{0}\right) \Theta^{\prime \prime}\left(l_{0}\right) \Theta^{(4)}\left(l_{0}\right) \\
&\left.+\frac{1}{504} \sin \Theta\left(l_{0}\right)\left[\Theta^{\prime \prime \prime}\left(l_{0}\right)\right]^{2}\right\}\left(l-l_{0}\right)^{7} \\
&-\left\{\frac{1}{384} \cos \Theta\left(l_{0}\right)\left[\Theta^{\prime \prime}\left(l_{0}\right)\right]^{2} \Theta^{\prime \prime \prime}\left(l_{0}\right)\right. \\
&\left.+\frac{1}{1152} \sin \Theta\left(l_{0}\right) \Theta^{\prime \prime \prime}\left(l_{0}\right) \Theta^{(4)}\left(l_{0}\right)\right\}\left(l-l_{0}\right)^{8} \\
&+\left\{\frac{1}{3456} \sin \Theta\left(l_{0}\right)\left[\Theta^{\prime \prime}\left(l_{0}\right)\right]^{4}\right. \\
&-\frac{1}{1296} \cos \Theta\left(l_{0}\right) \Theta^{\prime \prime}\left(l_{0}\right)\left[\Theta^{\prime \prime \prime}\left(l_{0}\right)\right]^{2} \\
& \quad-\frac{1}{1728} \cos \Theta\left(l_{0}\right)\left[\Theta^{\prime \prime}\left(l_{0}\right)\right]^{2} \Theta^{(4)}\left(l_{0}\right) \\
&\left.-\frac{1}{10368} \sin \Theta\left(l_{0}\right)\left[\Theta^{(4)}\left(l_{0}\right)\right]^{2}\right\}\left(l-l_{0}\right)^{9}+\cdots
\end{aligned}
$$

where

$$
\begin{aligned}
& \Theta\left(l_{0}\right)=k_{1} l_{0}+\frac{1}{l_{k}^{2}}\left(k_{2}-k_{1}\right) l_{0}^{3}-\frac{1}{2 l_{k}^{3}}\left(k_{2}-k_{1}\right) l_{0}^{4} \\
& \Theta^{\prime \prime}\left(l_{0}\right)=\frac{6}{l_{k}^{2}}\left(k_{2}-k_{1}\right) l_{0}-\frac{6}{l_{k}^{3}}\left(k_{2}-k_{1}\right) l_{0}^{2} \\
& \Theta^{\prime \prime \prime}\left(l_{0}\right)=\frac{6}{l_{k}^{2}}\left(k_{2}-k_{1}\right)-\frac{12}{l_{k}^{3}}\left(k_{2}-k_{1}\right) l_{0} \\
& \Theta^{(4)}\left(l_{0}\right)=-\frac{12}{l_{k}^{3}}\left(k_{2}-k_{1}\right) .
\end{aligned}
$$

Making use of parametric equations (31) and also (35) one can obtain a correct solution of the problem with diversified signs of curvature.

Value of the tangent slope at the end of the transition curve makes it possible to connect to it a curve of curvature $k_{2}$ satisfying simultaneously the tangency condition of both the curves. The above value is the same as in the case of linear curvature. It is defined by (25).

\section{Transition Curve of Curvature in Trigonometric Form}

5.1. Formulation of Curvature Equation. As before boundary conditions (26) and another differential equation are assumed.

$$
k^{(4)}(l)+\frac{\pi^{2}}{l_{k}^{2}} k^{\prime \prime}(l)=0
$$

As soon as the differential problem (26), (37) has been solved, a formula for curvature, is obtained

$$
k(l)=\frac{1}{2}\left(k_{1}+k_{2}\right)-\frac{1}{2}\left(k_{2}-k_{1}\right) \cos \frac{\pi}{l_{k}} l .
$$

The form of function $\Theta(l)$ determined by the use of (5) and (38) is as follows:

$$
\Theta(l)=\frac{1}{2}\left(k_{1}+k_{2}\right) l-\frac{1}{2}\left(k_{2}-k_{1}\right) \frac{l_{k}}{\pi} \sin \frac{\pi}{l_{k}} l .
$$

The length of the transition curve on railway routes follows from conditions:

$$
\begin{aligned}
& l_{k}^{\psi} \geq \frac{\pi}{2} \frac{v}{\psi_{\text {dop }}}\left|a_{2}-a_{1}\right|, \\
& l_{k}^{f} \geq \frac{\pi}{2} \frac{v}{f_{\text {dop }}}\left|h_{2}-h_{1}\right| .
\end{aligned}
$$

The assumed length of the curve must satisfy the condition (19).

5.2. Ordinates of Transition Curve Connecting Uniform Curvatures. After the expansion of functions $\cos \Theta(l)$ and $\sin \Theta(l)$ into Maclaurin series to be next integrated the following parametric equations are obtained:

$$
\begin{aligned}
x(l)= & \int \cos \Theta(l) d l \\
= & l-\frac{k_{1}^{2}}{6} l^{3}+\left[\frac{k_{1}^{4}}{120}-\frac{1}{60} \frac{\pi^{2}}{l_{k}^{2}} k_{1}\left(k_{2}-k_{1}\right)\right] l^{5} \\
- & {\left[\frac{k_{1}^{6}}{5040}-\frac{1}{504} \frac{\pi^{2}}{l_{k}^{2}} k_{1}^{3}\left(k_{2}-k_{1}\right)\right.} \\
& \left.-\frac{1}{1680} \frac{\pi^{4}}{l_{k}^{4}} k_{1}\left(k_{2}-k_{1}\right)-\frac{1}{2016} \frac{\pi^{4}}{l_{k}^{4}}\left(k_{2}-k_{1}\right)^{2}\right] l^{7} \\
+ & -\frac{k_{1}^{8}}{362880}-\frac{1}{12960} \frac{\pi^{2}}{l_{k}^{2}} k_{1}^{5}\left(k_{2}-k_{1}\right) \\
& -\frac{1}{12960} \frac{\pi^{4}}{l_{k}^{4}} k_{1}^{2}\left(k_{2}-k_{1}\right)^{2} \\
& \left.+\frac{1}{25920} \frac{\pi^{6}}{l_{k}^{6}}\left(k_{2}-k_{1}\right)^{2}\right] l^{9}+\ldots \\
& -\frac{1}{90720} \frac{\pi^{6}}{l_{k}^{6}} k_{1}\left(k_{2}-k_{1}\right)
\end{aligned}
$$




$$
\begin{aligned}
y(l)= & \int \sin \Theta(l) d l \\
= & \frac{1}{2} k_{1} l^{2}-\left[\frac{1}{24} k_{1}^{3}-\frac{1}{48} \frac{\pi^{2}}{l_{k}^{2}}\left(k_{2}-k_{1}\right)\right] l^{4} \\
+ & {\left[\frac{1}{720} k_{1}^{5}-\frac{1}{144} k_{1}^{2} \frac{\pi^{2}}{l_{k}^{2}}\left(k_{2}-k_{1}\right)\right.} \\
& \left.-\frac{1}{1440} \frac{\pi^{4}}{l_{k}^{4}}\left(k_{2}-k_{1}\right)\right] l^{6} \\
& +\frac{1}{230320} \frac{\pi_{1}^{7}}{l_{k}^{4}} k_{1}\left(k_{2}-\frac{1}{2304} k_{1}^{4} \frac{\pi^{2}}{l_{k}^{2}}\left(k_{2}-k_{1}\right)\right. \\
& \left.+\frac{1}{80640} \frac{1}{l_{k}^{6}}\left(k_{2}-k_{1}\right)\right] l^{8}+\cdots \\
l_{k}^{4} & k_{1}^{2}\left(k_{2}-k_{1}\right)
\end{aligned}
$$

Equations (41) for $k_{1}=0$ describe the curve known as cosinusoid which can be used to connect a straight with a circular arc.

5.3. Transition Curve Coordinates Connecting Inverse Curvatures. Near inverse arcs in the diagram of function $\Theta(l)$ there appears the extremum at point $l_{0}$, where

$$
\Theta^{\prime}\left(l_{0}\right)=k\left(l_{0}\right)=\frac{1}{2}\left(k_{1}+k_{2}\right)-\frac{1}{2}\left(k_{2}-k_{1}\right) \cos \frac{\pi}{l_{k}} l_{0}=0 .
$$

The transition curve parametric equations (41) are effective for $l \in\left\langle 0, l_{0}\right\rangle$. To determine value $l_{0}$ one must solve (42). The equation can be written in the form

$$
\cos \frac{\pi}{l_{k}} l_{0}=\frac{k_{1}+k_{2}}{k_{2}-k_{1}}
$$

It is easy to prove that for $\operatorname{sign} k_{1} \neq \operatorname{sign} k_{2}$ it is necessary to satisfy the condition $\left|k_{1}+k_{2}\right|<\left|k_{2}-k_{1}\right|$, thus $\mid\left(k_{1}+k_{2}\right) /\left(k_{2}-\right.$ $\left.k_{1}\right) \mid<1$. The formula for length $l_{0}$ is as follows:

$$
l_{0}=\frac{1}{\pi}\left(\arccos \frac{k_{1}+k_{2}}{k_{2}-k_{1}}\right) l_{k} .
$$

For $l \in\left(l_{0}, l_{k}\right\rangle$ functions $\cos \Theta(l)$ and $\sin \Theta(l)$ should be expanded into Taylor series. The integration provides the following equations:

$$
\begin{aligned}
& x(l)=\int \cos \Theta(l) d l \\
& =x\left(l_{0}\right)+\cos \Theta\left(l_{0}\right)\left(l-l_{0}\right) \\
& -\frac{1}{6} \sin \Theta\left(l_{0}\right) \Theta^{\prime \prime}\left(l_{0}\right)\left(l-l_{0}\right)^{3} \\
& -\frac{1}{24} \sin \Theta\left(l_{0}\right) \Theta^{\prime \prime \prime}\left(l_{0}\right) \cdot\left(l-l_{0}\right)^{4} \\
& -\left\{\frac{1}{40} \cos \Theta\left(l_{0}\right)\left[\Theta^{\prime \prime}\left(l_{0}\right)\right]^{2}\right. \\
& \left.+\frac{1}{120} \sin \Theta\left(l_{0}\right) \Theta^{(4)}\left(l_{0}\right)\right\}\left(l-l_{0}\right)^{5} \\
& -\left\{\frac{1}{72} \cos \Theta\left(l_{0}\right) \Theta^{\prime \prime}\left(l_{0}\right) \Theta^{\prime \prime \prime}\left(l_{0}\right)\right. \\
& \left.+\frac{1}{720} \sin \Theta\left(l_{0}\right) \Theta^{(5)}\left(l_{0}\right)\right\}\left(l-l_{0}\right)^{6} \\
& +\left\{\frac{1}{336} \sin \Theta\left(l_{0}\right)\left[\Theta^{\prime \prime}\left(l_{0}\right)\right]^{3}\right. \\
& -\frac{1}{336} \cos \Theta\left(l_{0}\right) \Theta^{\prime \prime}\left(l_{0}\right) \Theta^{(4)}\left(l_{0}\right) \\
& -\frac{1}{504} \cos \Theta\left(l_{0}\right)\left[\Theta^{\prime \prime \prime}\left(l_{0}\right)\right]^{2} \\
& \left.-\frac{1}{5040} \sin \Theta\left(l_{0}\right) \Theta^{(6)}\left(l_{0}\right)\right\}\left(l-l_{0}\right)^{7} \\
& +\left\{\frac{1}{384} \sin \Theta\left(l_{0}\right)\left[\Theta^{\prime \prime}\left(l_{0}\right)\right]^{2} \Theta^{\prime \prime \prime}\left(l_{0}\right)\right. \\
& -\frac{1}{1920} \cos \Theta\left(l_{0}\right) \Theta^{\prime \prime}(l) \Theta^{(5)}(l) \\
& -\frac{1}{1152} \cos \Theta\left(l_{0}\right) \Theta^{\prime \prime \prime}\left(l_{0}\right) \Theta^{(4)}\left(l_{0}\right) \\
& \left.-\frac{1}{40320} \sin \Theta\left(l_{0}\right) \Theta^{(7)}\left(l_{0}\right)\right\}\left(l-l_{0}\right)^{8} \\
& +\left\{\frac{1}{3456} \cos \Theta\left(l_{0}\right)\left[\Theta^{\prime \prime}\left(l_{0}\right)\right]^{4}\right. \\
& +\frac{1}{1296} \sin \Theta\left(l_{0}\right) \Theta^{\prime \prime}\left(l_{0}\right)\left[\Theta^{\prime \prime \prime}\left(l_{0}\right)\right]^{2} \\
& +\frac{1}{1728} \sin \Theta\left(l_{0}\right)\left[\Theta^{\prime \prime}\left(l_{0}\right)\right]^{2} \Theta^{(4)}\left(l_{0}\right) \\
& -\frac{1}{12960} \cos \Theta\left(l_{0}\right) \Theta^{\prime \prime}\left(l_{0}\right) \Theta^{(6)}\left(l_{0}\right) \\
& -\frac{1}{6480} \cos \Theta\left(l_{0}\right) \Theta^{\prime \prime \prime}\left(l_{0}\right) \Theta^{(5)}\left(l_{0}\right) \\
& -\frac{1}{10368} \cos \Theta\left(l_{0}\right)\left[\Theta^{(4)}\left(l_{0}\right)\right]^{2} \\
& \left.-\frac{1}{362880} \sin \Theta\left(l_{0}\right) \Theta^{(8)}\left(l_{0}\right)\right\}\left(l-l_{0}\right)^{9}+\cdots
\end{aligned}
$$




$$
\begin{aligned}
& y(l)=\int \sin \Theta(l) d l \\
& =y\left(l_{0}\right)+\sin \Theta\left(l_{0}\right)\left(l-l_{0}\right) \\
& +\frac{1}{6} \cos \Theta\left(l_{0}\right) \Theta^{\prime \prime}\left(l_{0}\right)\left(l-l_{0}\right)^{3} \\
& +\frac{1}{24} \cos \Theta\left(l_{0}\right) \Theta^{\prime \prime \prime}\left(l_{0}\right) \cdot\left(l-l_{0}\right)^{4} \\
& -\left\{\frac{1}{40} \sin \Theta\left(l_{0}\right)\left[\Theta^{\prime \prime}\left(l_{0}\right)\right]^{2}\right. \\
& \left.-\frac{1}{120} \cos \Theta\left(l_{0}\right) \Theta^{(4)}\left(l_{0}\right)\right\}\left(l-l_{0}\right)^{5} \\
& -\left\{\frac{1}{72} \sin \Theta\left(l_{0}\right) Q^{\prime \prime}\left(l_{0}\right) \Theta^{\prime \prime \prime}\left(l_{0}\right)\right. \\
& \left.-\frac{1}{720} \cos \Theta\left(l_{0}\right) \Theta^{(5)}\left(l_{0}\right)\right\}\left(l-l_{0}\right)^{6} \\
& -\left\{\frac{1}{336} \cos \Theta\left(l_{0}\right)\left[\Theta^{\prime \prime}\left(l_{0}\right)\right]^{3}\right. \\
& +\frac{1}{336} \sin \Theta\left(l_{0}\right) \Theta^{\prime \prime}\left(l_{0}\right) \Theta^{(4)}\left(l_{0}\right) \\
& +\frac{1}{504} \sin \Theta\left(l_{0}\right)\left[\Theta^{\prime \prime \prime}\left(l_{0}\right)\right]^{2} \\
& \left.-\frac{1}{5040} \cos \Theta\left(l_{0}\right) \Theta^{(6)}\left(l_{0}\right)\right\}\left(l-l_{0}\right)^{7} \\
& \Theta\left(l_{0}\right)=\frac{1}{2}\left(k_{1}+k_{2}\right) l_{0} \pm \frac{l_{k}}{\pi} \sqrt{-k_{1} k_{2}} \\
& \Theta^{\prime \prime}\left(l_{0}\right)=\mp \frac{\pi}{l_{k}} \sqrt{-k_{1} k_{2}} \\
& \Theta^{\prime \prime}\left(l_{0}\right)=\mp \frac{\pi}{l_{k}} \sqrt{-k_{1} k_{2}} \\
& \Theta^{\prime \prime \prime}\left(l_{0}\right)=\frac{1}{2} \frac{\pi^{2}}{l_{k}^{2}}\left(k_{1}+k_{2}\right) \\
& \Theta^{(4)}\left(l_{0}\right)= \pm \frac{\pi^{3}}{l_{k}^{3}} \sqrt{-k_{1} k_{2}} \\
& \Theta^{(5)}\left(l_{0}\right)=-\frac{1}{2} \frac{\pi^{4}}{l_{k}^{4}}\left(k_{1}+k_{2}\right) \\
& \Theta^{(6)}\left(l_{0}\right)=\mp \frac{\pi^{5}}{l_{k}^{5}} \sqrt{-k_{1} k_{2}} \\
& \Theta^{(7)}\left(l_{0}\right)=\frac{1}{2} \frac{\pi^{6}}{l_{k}^{6}}\left(k_{1}+k_{2}\right) \\
& \Theta^{(8)}\left(l_{0}\right)= \pm \frac{\pi^{7}}{l_{k}^{7}} \sqrt{-k_{1} k_{2}} \text {. }
\end{aligned}
$$$$
-\left\{\frac{1}{384} \cos \Theta\left(l_{0}\right)\left[\Theta^{\prime \prime}\left(l_{0}\right)\right]^{2} \Theta^{\prime \prime \prime}\left(l_{0}\right)\right.
$$$$
+\frac{1}{1920} \sin \Theta\left(l_{0}\right) \Theta^{\prime \prime}\left(l_{0}\right) \Theta^{(5)}\left(l_{0}\right)
$$$$
+\frac{1}{1152} \sin \Theta\left(l_{0}\right) \Theta^{\prime \prime \prime}\left(l_{0}\right) \Theta^{(4)}\left(l_{0}\right)
$$$$
\left.-\frac{1}{40320} \cos \Theta\left(l_{0}\right) \Theta^{(7)}\left(l_{0}\right)\right\}\left(l-l_{0}\right)^{8}
$$$$
+\left\{\frac{1}{3456} \sin \Theta\left(l_{0}\right)\left[\Theta^{\prime \prime}\left(l_{0}\right)\right]^{4}\right.
$$$$
-\frac{1}{1296} \cos \Theta\left(l_{0}\right) \Theta^{\prime \prime}\left(l_{0}\right)\left[\Theta^{\prime \prime \prime}\left(l_{0}\right)\right]^{2}
$$$$
-\frac{1}{1728} \cos \Theta\left(l_{0}\right)\left[\Theta^{\prime \prime}\left(l_{0}\right)\right]^{2} \Theta^{(4)}\left(l_{0}\right)
$$$$
-\frac{1}{12960} \sin Q\left(l_{0}\right) Q^{\prime \prime}\left(l_{0}\right) Q^{(6)}\left(l_{0}\right)
$$$$
-\frac{1}{6480} \sin Q\left(l_{0}\right) Q^{\prime \prime \prime}\left(l_{0}\right) Q^{(5)}\left(l_{0}\right)
$$$$
-\frac{1}{10368} \sin \Theta\left(l_{0}\right)\left[\Theta^{(4)}\left(l_{0}\right)\right]^{2}
$$$$
\left.+\frac{1}{362880} \cos \Theta\left(l_{0}\right) \Theta^{(8)}\left(l_{0}\right)\right\}\left(l-l_{0}\right)^{9}+\cdots,
$$

In expressions with double signs \pm and $\mp$, the upper one corresponds to the case $k_{1}>0, k_{2}<0$, while the lower one refers to $k_{1}<0, k_{2}>0$.

The value of tangent slope at the end of the transition curve makes it possible to connect to it a curve of curvature $k_{2}$ satisfying the tangency condition of both the curves. The value is the same as in the previous cases. It is determined by (25).

\section{Comparison of Horizontal Ordinates of Determined Transition Curves}

6.1. The Shaping of Horizontal Ordinates of Comparable Curves. If the analysis is carried out for railway routes, then the necessity of meeting kinematic conditions (10) and (11) will require a diversification of the lengths of the compared with each other curves (using the same speed of trains). Moreover, it is necessary to individually consider cases of connecting homogeneous curvatures $\left(\operatorname{sign} k_{1}=\operatorname{sign} k_{2}\right)$ and inverse $\operatorname{arcs}\left(\operatorname{sign} k_{1} \neq \operatorname{sign} k_{2}\right)$.

It is assumed that at joints of consistent arcs with radii $R_{1}=1200 \mathrm{~m}$ and $R_{2}=700 \mathrm{~m}$ an attempt is made to attain a speed $v=110 \mathrm{~km} / \mathrm{h}$. Hence it is necessary to employ on arcs superelevation of $h_{1}=80 \mathrm{~mm}$ and $h_{2}=115 \mathrm{~mm}$, respectively. The retention of the same kinematic parameters can provide diversified lengths of the transition curve:

(i) for a curve of linear curvature $l_{k}=40 \mathrm{~m}$,

(ii) for a curve of polynomial curvature $l_{k}=60 \mathrm{~m}$,

(iii) for a curve of trigonometric curvature $l_{k}=62,832 \mathrm{~m}$. 
The above length relations result directly from the equations given at Sections 3.2, 4.1, and 5.1.

Figure 5 gives diagrams of horizontal ordinates for compared transition curves of positive and negative curvature values. As seen the curvature ordinates of linear curvature evidently deviate from the ordinates of other curves. In turn, curves of polynomial and trigonometric curvature are in principle consistent. A difference can only be noted in the final area. And so, the class of the function describing the curvature plays here a significant part: with respect to a curve of linear curvature it is a function of class $\mathrm{C}^{0}$, while regarding the other two curves it is a function of class $C^{1}$.

At the joint of inverse arcs of radii $R_{1}=1200 \mathrm{~m}$ and $R_{2}=$ $700 \mathrm{~m}$ an attempt is made to reach the speed of $v=90 \mathrm{~km} / \mathrm{h}$. Hence it follows that it is necessary to apply superelevation along arcs attaining $h_{1}=20 \mathrm{~mm}$ and $h_{2}=45 \mathrm{~mm}$, respectively. By the use the same kinematic parameters it is possible to obtain the following lengths of the transition curve:

(i) for a curve of linear curvature $l_{k}=60 \mathrm{~m}$,

(ii) for a curve of polynomial curvature $l_{k}=90 \mathrm{~m}$,

(iii) for a curve of trigonometric curvature $l_{k}=94,248 \mathrm{~m}$.

Figure 6 presents diagrams of horizontal ordinates of compared transition curves for positive and negative values of curvature.

As can be seen, like in the case of consistent arcs, the curve ordinates of linear curvature deviate significantly from the ordinates of the remaining curves, but the shape of these curves also differs one from the other. The class of function describing the curvature is still important. However, in the range of the same class various geometric solutions are possible.

6.2. Negotiation of Transition Curves in a Geometric System. The transition curves do not appear alone in a geometric system but must be connected with some arcs in the neighborhood. If the input arc is circular, then its equation is as follows:

(i) for $k_{1}>0$

$$
y(x)=R_{1}-\sqrt{R_{1}^{2}-x^{2}},
$$

(ii) for $k_{1}<0$

$$
y(x)=-\left(R_{1}-\sqrt{R_{1}^{2}-x^{2}}\right),
$$

where $x<0$.

However, if the output arc is also a circular arc, then it is expressed in the following equation:

(i) for $k_{2}>0$

$$
\begin{gathered}
y(x)=y_{S}-\sqrt{R_{2}^{2}-\left(x_{S}-x\right)^{2}} \\
x_{S}=x_{K}-\frac{s_{K}}{\sqrt{1+s_{K}^{2}}} R_{2}, \quad y_{S}=y_{K}+\frac{1}{\sqrt{1+s_{K}^{2}}} R_{2},
\end{gathered}
$$

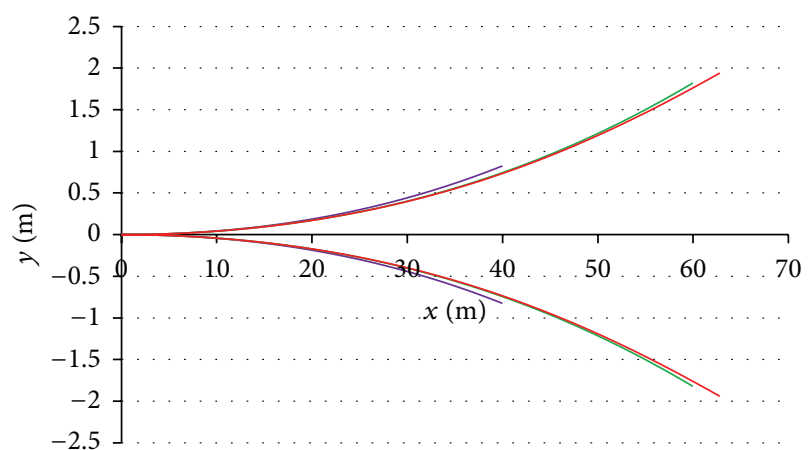

FIgURE 5: Diagrams of horizontal ordinates of curves joining consistent arcs for positive and negative values of curvature (using different horizontal and vertical scales): curve of linear curvature: line in violet colour, curve of polynomial curvature in green colour, and curve of trigonometric curvature in red colour.

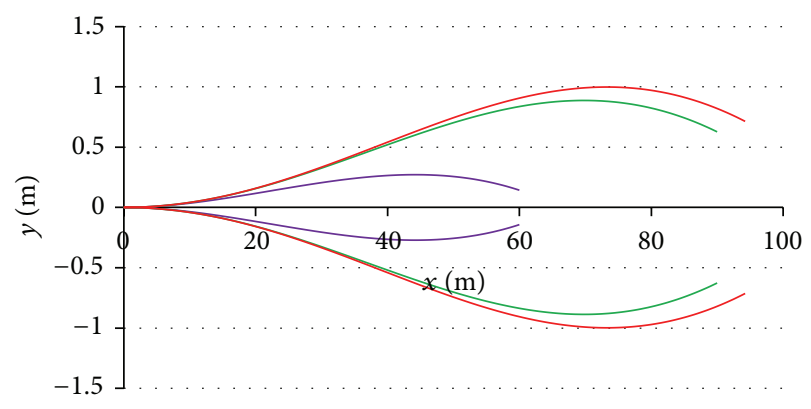

FIGURE 6: Diagrams of horizontal ordinates of curves connecting inverse arcs for positive and negative values of curvature (using different horizontal and vertical scales): the curve of linear curvature is in violet color, the curve of polynomial curvature is in green color, and the curve of trigonometric curvature is in red color.

(ii) for $k_{2}<0$

$$
\begin{gathered}
y(x)=y_{S}+\sqrt{R_{2}^{2}-\left(x_{S}-x\right)^{2}} \\
x_{S}=x_{K}+\frac{s_{K}}{\sqrt{1+s_{K}^{2}}} R_{2}, \quad y_{S}=y_{K}-\frac{1}{\sqrt{1+s_{K}^{2}}} R_{2},
\end{gathered}
$$

where $x>x_{K}$.

In (49) and (50) $x_{K}$ denotes the abscissae of the transition curve end, $y_{K}$ is the ordinate of the transition curve end, while $s_{K}$ is the value of tangent at the end of the transition curve. Values for numerical examples under consideration are given in Table 1.

Figures 7 and 8 illustrate the geometric systems made up of two circular arcs of consistent curvature, connected with various types of transition curves. As seen the application of a given type of transition curve leads to an adequate position of the output circular arc. The position of this arc by the use of a linear transition curve clearly deviates from such cases where advantage is taken of curves with polynomial and trigonometrical curvature. With regard to the latter a mutual position of the circular arc varies insignificantly. 
TABLE 1: Comparison of values $x_{K}, y_{K}$, and $s_{K}$ for numerical examples analyzed.

\begin{tabular}{|c|c|c|c|c|c|}
\hline Curve & $l_{k}[\mathrm{~m}]$ & $\Theta\left(l_{k}\right)[\mathrm{rad}]$ & $s_{K}$ & $x_{K}[\mathrm{~m}]$ & $y_{K}[\mathrm{~m}]$ \\
\hline \multicolumn{6}{|c|}{ Case $1: k_{1}=1 / 1200 \mathrm{rad} / \mathrm{m}$ and $k_{2}=1 / 700 \mathrm{rad} / \mathrm{m}$} \\
\hline Curve $C^{0}$ & 40 & 0,045238 & 0,045269 & 39,988 & 0,825 \\
\hline Curve $C^{1}(p)$ & 60 & 0,067857 & 0,067961 & 59,963 & 1,821 \\
\hline Curve $C^{1}(t)$ & 62,832 & 0,071060 & 0,071180 & 62,796 & 1,940 \\
\hline \multicolumn{6}{|c|}{ Case $2: k_{1}=1 / 1200 \mathrm{rad} / \mathrm{m}$ and $k_{2}=-1 / 700 \mathrm{rad} / \mathrm{m}$} \\
\hline Curve $\mathrm{C}^{0}$ & 60 & $-0,017857$ & $-0,017859$ & 59,999 & 0,143 \\
\hline Curve $C^{1}(p)$ & 90 & $-0,026786$ & $-0,026792$ & 89,991 & 0,627 \\
\hline Curve $C^{1}(t)$ & 94,248 & $-0,028050$ & $-0,028057$ & 94,238 & 0,714 \\
\hline \multicolumn{6}{|c|}{ Case 3: $k_{1}=-1 / 1200 \mathrm{rad} / \mathrm{m}$ and $k_{2}=1 / 700 \mathrm{rad} / \mathrm{m}$} \\
\hline Curve $C^{0}$ & 60 & 0,01786 & 0,017859 & 59,999 & $-0,143$ \\
\hline Curve $C^{1}(p)$ & 90 & 0,026786 & 0,026792 & 89,991 & $-0,627$ \\
\hline Curve $C^{1}(t)$ & 94,248 & 0,028050 & 0,028057 & 94,238 & $-0,714$ \\
\hline \multicolumn{6}{|c|}{ Case $4: k_{1}=-1 / 1200 \mathrm{rad} / \mathrm{m}$ and $k_{2}=-1 / 700 \mathrm{rad} / \mathrm{m}$} \\
\hline Curve $\mathrm{C}^{0}$ & 40 & $-0,045238$ & $-0,045269$ & 39,988 & $-0,825$ \\
\hline Curve $C^{1}(p)$ & 60 & $-0,067857$ & $-0,067961$ & 59,969 & $-1,821$ \\
\hline Curve $C^{1}(t)$ & 62,832 & $-0,071060$ & $-0,071180$ & 62,976 & $-1,940$ \\
\hline
\end{tabular}

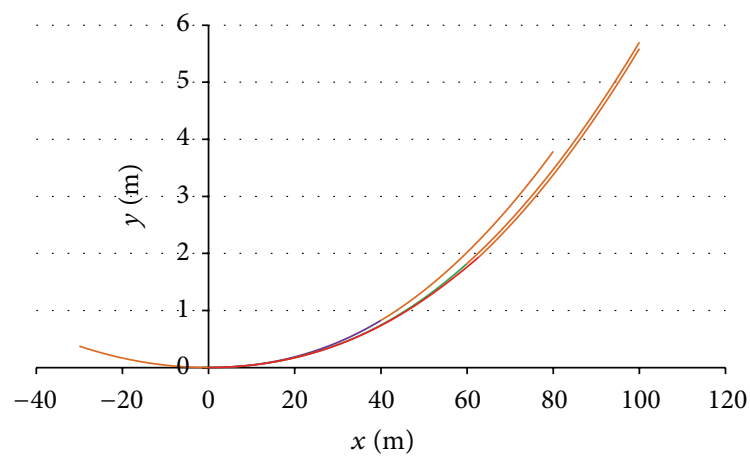

FIGURE 7: Examples of geometric systems consisting of two circular arcs with consistent curvature (brown line) connected with different transition curves, for positive values of curvature (using different horizontal and vertical scales): curve of linear curvature in violet line, curve of polynomial curvature in green, and curve of trigonometric curvature in red.

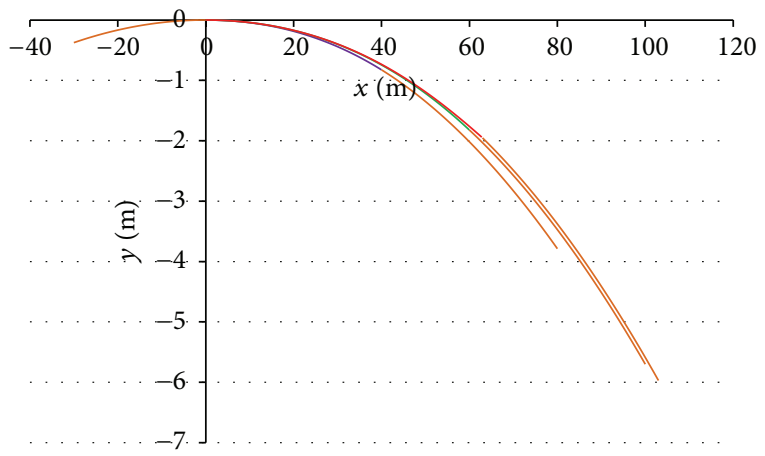

FIGURE 8: Examples of geometric systems consisting of two circular arcs with consistent curvature (brown line) connected with different transition curves, for negative values of curvature (using different horizontal and vertical scales): curve of linear curvature in violet line, curve of polynomial curvature in green, and curve of trigonometric curvature in red.

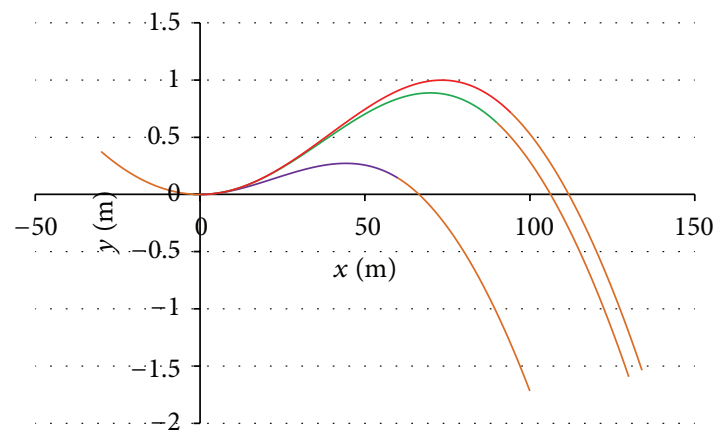

FIGURE 9: Examples of geometric systems consisting of two inverse circular arcs (brown line), connected with different types of transition curves, for positive values of input curvature (using different horizontal and vertical scales): curve of linear curvature in violet line, curve of polynomial curvature in green, and curve of trigonometric curvature in red.

Figures 9 and 10 illustrate geometric systems made up of two inverse circular arcs connected with various types of transition curves.

Here the layout of the output circular arc is much more diversified. The position of the arc obtained by the application of the transition curve of linear curvature diverts by several dozen meters from situations where curves of polynomial and trigonometric curvature have been used. The difference for curves of polynomial and trigonometric curvature attains, in the numerical example analyzed, an order of several meters.

The presented examples are taken, in the very nature of things, at random. They reveal wide possibilities for the presented analytical method in modelling geometric systems. Its application makes it possible to generate diversified layout of the communication routes adapted to the field conditions and other circumstances. Simultaneously the method ensures correctness of the obtained solution in view of the kinematic parameters magnitudes in force. 


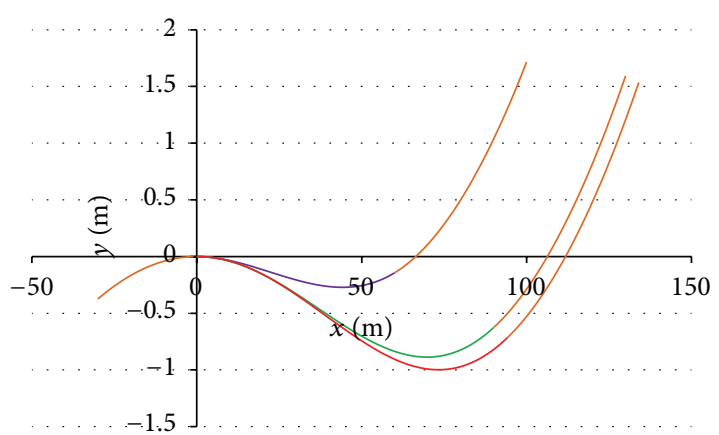

FIGURE 10: Examples of geometric systems consisting of two inverse circular arcs (brown line), connected with different types of transition curves, for negative values of input curvature (using different horizontal and vertical scales): curve of linear curvature in violet line, curve of polynomial curvature in green, and curve of trigonometric curvature in red.

\section{Conclusions}

The presented universal method of modelling geometrical system of a communication route is based on the determination of an adequate curvature equation by using differential equations. The technique enables us to design connections of various geometrical elements of the route indicating diversification in its curvature. It is characterized by an analytical way of recording certain functions, and moreover it is possible to specify in advance the class of these functions.

The sought-after connection is recorded in the form of parametric equations $x(l)$ and $y(l)$, where parameter $l$ is the position of a given point along the length of the curve. The determination of $x(l)$ and $y(l)$ needs the expansion of integrand functions into Maclaurin series and with regard to inverse curvatures into Taylor series.

The determination of coordinates of an adequate transition curve in rectangular coordinate system $x, y$ makes it possible for an easy transfer of it to Gauss-Kruger $(X, Y)$ conformal coordinates and its subsequent layout in field making use of the satellite measuring technique GNSS.

The method can be applied to identify various types of transition curves. The paper gives examples of solving the problem involving linear change of curvature and the curvature in polynomial and trigonometric form.

The performed comparative analysis reveals a wide range of possibilities which are offered by the presented design method. Its application enables us to generate diversified layout of the route. It simultaneously ensures a complete control of correctness of the obtained solutions in view of satisfying the geometric and kinematic conditions.

The presented method of modelling curvature has a universal character. It can be used for vehicular roads and railway lines, as well. In the case of railway lines there arises an additional possibility of modelling, in a similar way, the superelevation ramp understood here as a determined difference in the height of the rail courses.

\section{Conflict of Interests}

The author declares that he has no conflict of interests regarding the publication of this paper.

\section{References}

[1] W. Koc and C. Specht, "Application of the Polish active GNSS geodetic network for surveying and design of the railroad," in Proceedings of the 1st International Conference on Road and Rail Infrastructure (CETRA '10), pp. 757-762, May 2010.

[2] P. Lipar, M. Lakner, T. Maher, and M. Žura, "Estimation of road centerline curvature from raw GPS data," Baltic Journal of Road and Bridge Engineering, vol. 6, no. 3, pp. 163-168, 2011.

[3] L. Tasci and N. Kuloglu, "Investigation of a new transition curve," The Baltic Journal of Road and Bridge Engineering, vol. 6, no. 1, pp. 23-29, 2011.

[4] H.-H. Cai and G.-J. Wang, "A new method in highway route design: joining circular arcs by a single C-Bézier curve with shape parameter," Journal of Zhejiang University: Science A, vol. 10, no. 4, pp. 562-569, 2009.

[5] S. Dimulyo, Z. Habib, and M. Sakai, "Fair cubic transition between two circles with one circle inside or tangent to the other," Numerical Algorithms, vol. 51, no. 4, pp. 461-476, 2009.

[6] G. Farin, "Class A Bézier curves," Computer Aided Geometric Design, vol. 23, no. 7, pp. 573-581, 2006.

[7] Z. Habib and M. Sakai, " $\mathrm{G}^{2}$ pythagorean hodograph quintic transition between two circles with shape control," Computer Aided Geometric Design, vol. 24, no. 5, pp. 252-266, 2007.

[8] G. Harary and A. Tal, “The natural 3D spiral," Computer Graphics Forum, vol. 30, no. 2, pp. 237-246, 2011.

[9] K. T. Miura, "A general equation of aesthetic curves and its selfaffinity," Computer-Aided Design and Applications, vol. 3, no. 14, pp. 457-464, 2006.

[10] S. H. Yahaya, M. S. Salleh, and J. M. Ali, "Spur gear design with an S-shaped transition curve application using mathematica and CAD tools," in Proceedings of the International Conference on Computer Technology and Development (ICCTD '09), pp. 273-276, Dubai, United Arab Emirates, November 2009.

[11] N. Yoshida and T. Saito, "Quasi-aesthetic curves in rational cubic Bézier forms," Computer-Aided Design and Applications, vol. 4, no. 1-6, pp. 477-486, 2007.

[12] R. Ziatdinov, N. Yoshida, and T.-W. Kim, "Analytic parametric equations of log-aesthetic curves in terms of incomplete gamma functions," Computer Aided Geometric Design, vol. 29, no. 2, pp. 129-140, 2012.

[13] R. J. Grabowski, "Smooth curvilinear transitions along vehicular roads and railway lines," Science Papers of AGH University of Science and Technology, no. 82, Cracow, Poland, 1984 (Polish).

[14] L. T. Klauder, "Track transition curve geometry based on gegenbauer polynomials," in Proceedings of the 6th International Conference on Railway Engineering, May 2003.

[15] W. Koc, Elements of Design Theory on Track Systems, Gdansk University of Technology, Gdansk, Poland, 2004, (Polish).

[16] E. Mieloszyk and W. Koc, "General dynamic method for determining transition curve equations," Rail InternationalSchienen der Welt, vol. 10, pp. 32-40, 1991.

[17] B. Kufver, "Realigning railway in track renewals-linear versus S-shaped superelevation ramps," in Proceedings of the 2nd International Conference on Railway Engineering, May 1999.

[18] W. Koc and E. Mieloszyk, "Comparative analysis of selected transition curves by the use of dynamic model," Archives of Civil Engineering, vol. 33, no. 2, pp. 239-261, 1987 (Polish).

[19] S. Gleason and D. Gebre-Egziabher, GNSS Applications and Methods, Artech House Publishers, Norwood, NJ, USA, 2009. 
[20] B. Hoffmann-Wellenhof, H. Lichtenegger, and E. Wasle, GNSS Global Navigation Satellite Systems-GPS, GLONASS, Galileo \& More, Springer, Wien, Austria, 2008.

[21] P. Misra and P. Enge, Global Positioning System: Signals, Measurements, and Performance, Ganga-Jamuna Press, Lincoln, Mass, USA, 2012.

[22] C. Specht, "Accuracy and coverage of the modernized Polish Maritime differential GPS system," Advances in Space Research, vol. 47, no. 2, pp. 221-228, 2011.

[23] W. Koc and K. Palikowska, "Dynamic properties evaluation of the selected methods of joining route segments with different curvature," Technika Transportu Szynowego, vol. 9, pp. 17851807, 2012 (Polish). 


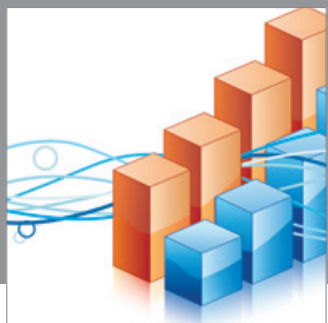

Advances in

Operations Research

mansans

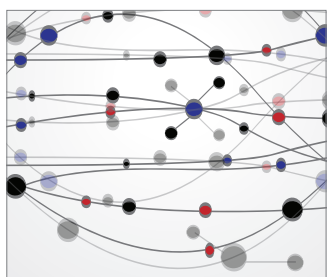

The Scientific World Journal
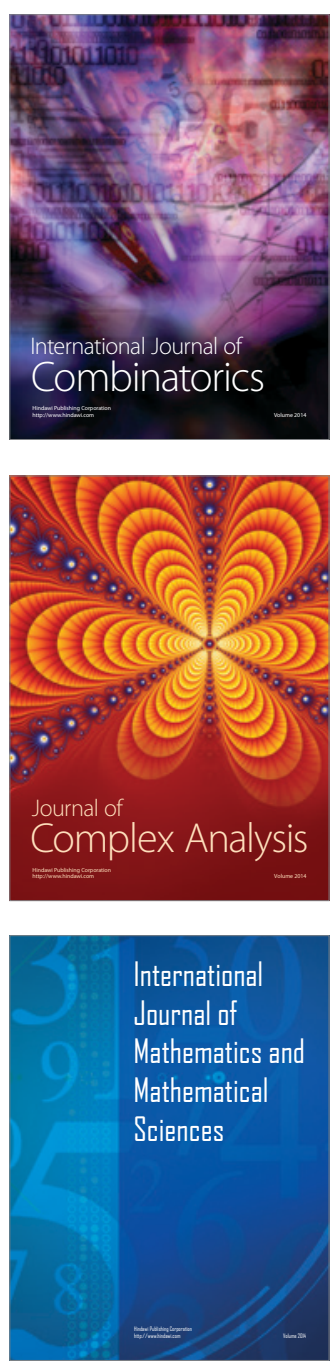
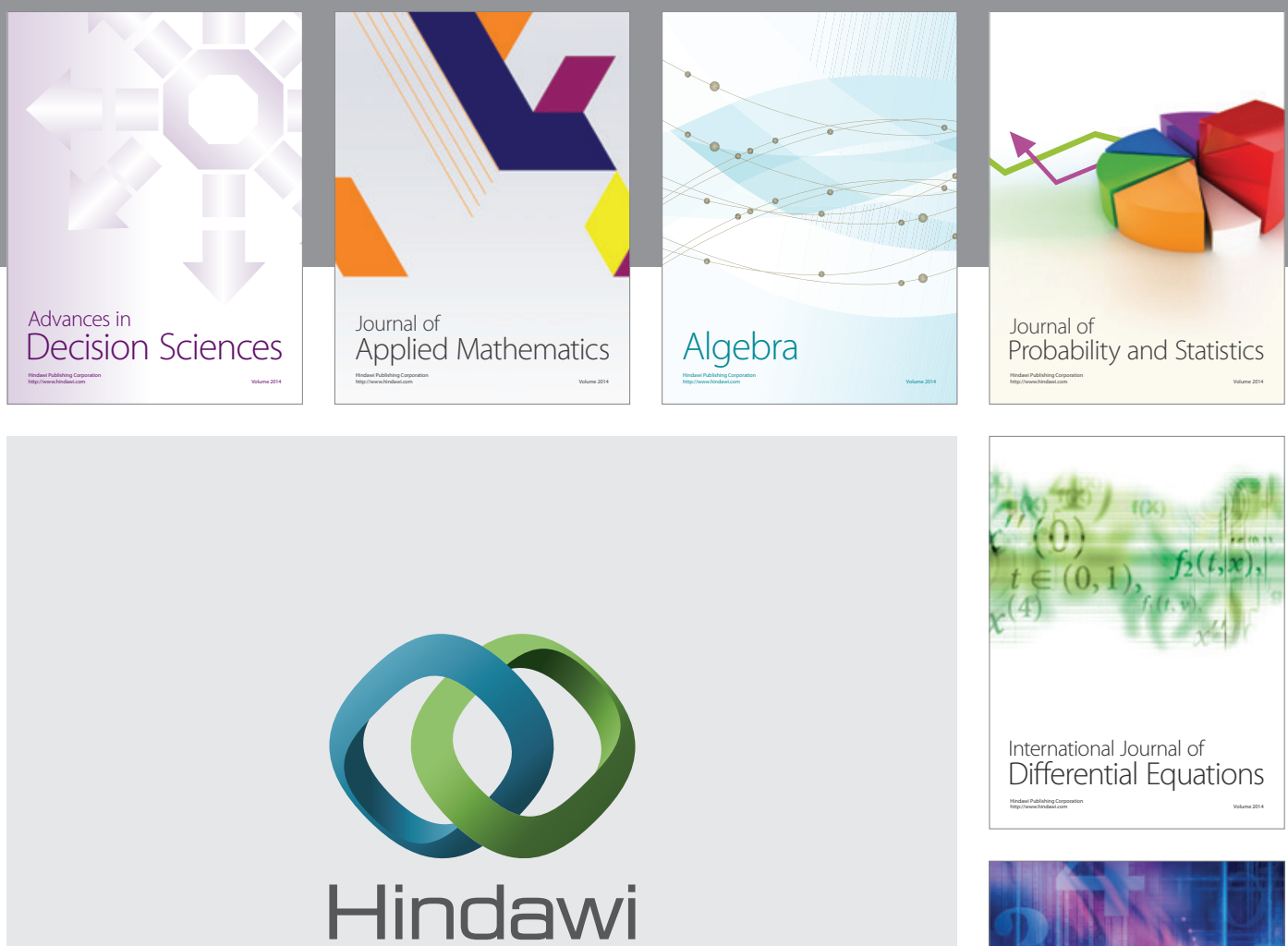

Submit your manuscripts at http://www.hindawi.com
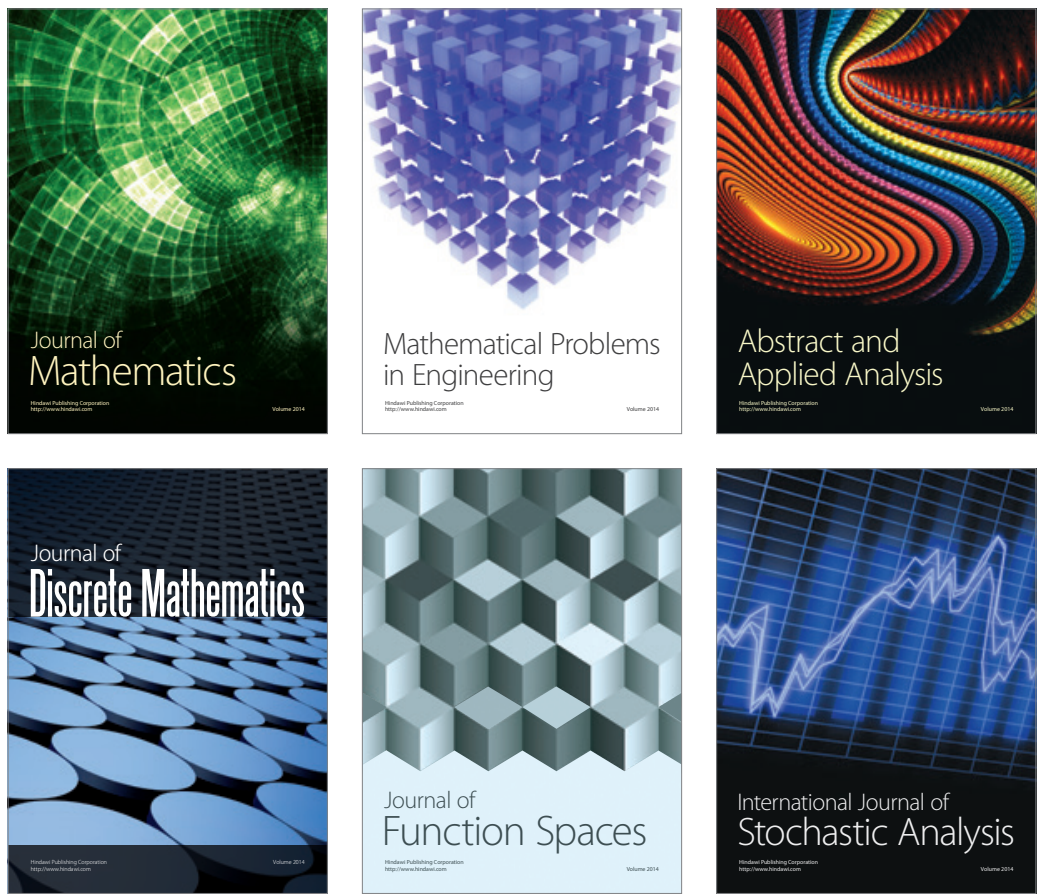

Journal of

Function Spaces

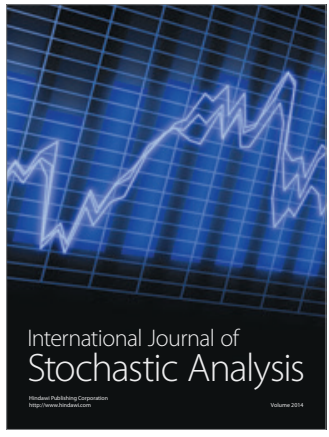

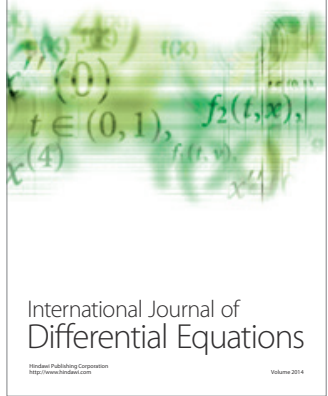
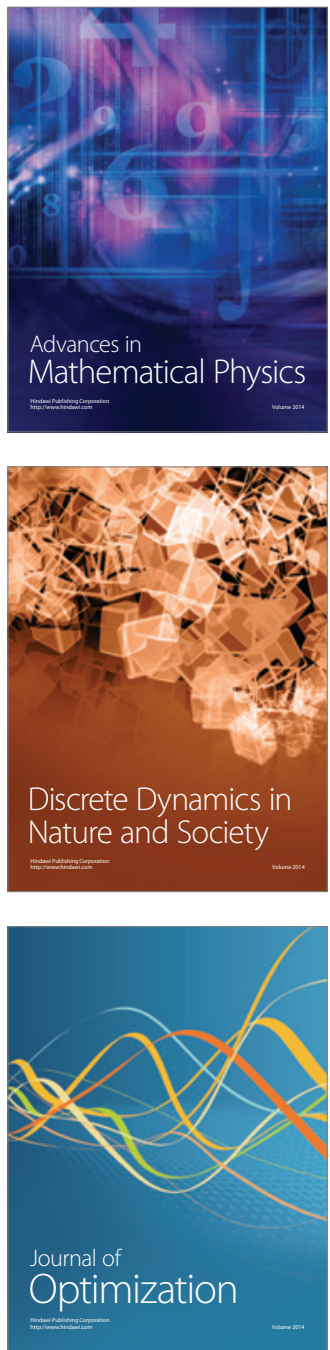